JOURNAL OF

APPLIED

CRYSTALLOGRAPHY

ISSN 1600-5767

Accepted 9 January 2019

Edited by S. Sasaki, Tokyo Institute of

Technology, Yokohama, Japan

Keywords: lattice; sublattice; superlattice; substructure; superstructure; crystallographic orbit; Wyckoff positions; subperiodic groups.

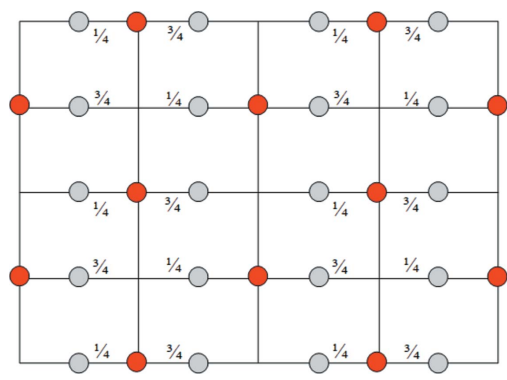

C 2019 International Union of Crystallography

\section{Lattice versus structure, dimensionality versus periodicity: a crystallographic Babel?}

\author{
Massimo Nespolo* \\ Université de Lorraine, CNRS, CRM2, Nancy, France. *Correspondence e-mail: massimo.nespolo@univ-lorraine.fr
}

In this article, several mistakes occurring in the description of crystal structures are pointed out. (1) The term 'lattice' is often used instead of 'structure'. (2) The two terms 'superlattice' and 'sublattice' are incorrectly interchanged. (3) Substructures composed of a subset of the crystallographic orbits composing the structure are incorrectly called 'sublattices'. (4) Subperiodic structures are incorrectly referred to as 'low-dimensional' structures.

\section{Introduction}

A huge literature in solid state physics and chemistry, metallurgy, materials science, mineralogy, and related fields appears every year where unfortunately imprecise and often incorrect crystallographic terminology is adopted. Quite surprisingly, among the most misused terms we find the very basic notion of lattice, which seems to represent an unexpected trap in the literature dealing with condensed matter. Disputable terms like 'lattice defects' (actually meaning structural defects: e.g. Devi et al., 2017) have become entrenched in the literature like weeds in a field; worse than that, completely nonsensical expressions like 'diamond lattice' (e.g. Lundow \& Campbell, 2018) or 'sphalerite lattice' (e.g. Sarvaramini et al., 2016) pass through the review process and go to press in highly respectable publications. Other examples of incorrect uses concern the confusion between a 'superlattice' and a 'sublattice', often used contrary to their definition and meaning; the use of 'sublattice' to indicate part of a structure built on some of the atoms composing the full structure; and the incorrect description as a 'low-dimensional structure' of what is actually a three-dimensional structure which is periodic in a subspace. Considering how often one can nowadays spot these mistakes in the literature, we think it is time to remind the reader of some fundamental definitions and to provide some simple directions to avoid falling into these terminological traps. For that, we need, however, to review a few terms and definitions.

(i) An ideal crystal is a crystal which does not present any static (inclusions, dislocations, precipitates etc.) or dynamic (phonons) defects and whose atomic distribution is perfectly periodic in a space with a sufficient number of dimensions.

(ii) The infinite set of atoms obtained by applying the operations of a space group to a single atom in the asymmetric unit constitutes a crystallographic orbit. There is therefore a 1:1 correspondence between the number of atoms in the asymmetric unit and the number of crystallographic orbits that build up the crystal structure, but each crystallographic orbit is an infinite set. The symmetry group of a crystallographic orbit is usually called its eigensymmetry and indicated as $\mathscr{E}$. 
(iii) The multiplicity of a Wyckoff position is the number of atomic positions within the unit cell that belong to a single crystallographic orbit corresponding to that position.

(iv) The site-symmetry group of an atomic position ('site') is the subgroup of the space group which leaves invariant ("fixes; 'does not move') the coordinates of that site. A site-symmetry group is isomorphic to one of the 32 types of crystallographic point groups, but its symmetry operations contain also translational components which reflect the position in space of the corresponding symmetry elements.

\section{Lattice, sublattice and superlattice}

The concept of lattice can be introduced in a very general but rather abstract way [see e.g. Conway \& Sloane (1998)]. Here we target (ideal) crystal structures and their translation lattices; accordingly, our definition is potentially less general but more oriented to the practical crystallographer.

The atomic distribution within a crystal is called the crystal structure. If we neglect all possible defects in the structure, we get what is known as an ideal crystal structure, whose periodicity defines the (Bravais) lattice of the crystal. In other words, the lattice of a crystal structure can be seen as a collection of vectors expressed as a linear combination of $n$ linearly independent vectors. Here $n$ is the number of dimensions of the space along which the structure is periodic and does not necessarily coincide with the number of dimensions $N$ of the space. Depending on whether $n=N$ or $n<$ $N$, one speaks of periodic or subperiodic structure (nonperiodic structure being the limiting case when $n=0$ ). Graphene is a well known example of subperiodic structure, in particular diperiodic structure $(n=2, N=3)$. Modular structures are structures built from building blocks (modules) stacked one with respect to the others in a regular and ordered way (Nespolo et al., 2004). The modules (layers, rods, bricks) are another example of subperiodic structures. The symmetry of the whole structure is periodic in the whole crystal space and is described by an $N$-dimensional space group $\mathcal{G}_{N}^{N}(N \geq 3)$. Its modules are periodic in a subspace and their symmetry is described by an $N$-dimensional subperiodic group $\mathcal{G}_{n}^{N}(N \geq 3)$ ( $\mathcal{G}_{0}^{N}$ point group, $\mathcal{G}_{1}^{3}$ rod group, $\mathcal{G}_{2}^{3}$ layer group; groups $\mathcal{G}_{0<n<N}^{N>3}$ do not have a special name) ${ }^{\mathbf{1}}$ (Kopský \& Litvin, 2010). For $n=$ 0 , the subperiodic group degenerates into an $\mathrm{N}$-dimensional point group $G_{0}^{N}$; in this case the module is non-periodic and does not possesses a lattice.

The space group $\mathcal{G}_{3}^{3}$ of a crystal structure, in the following shortened to $\mathcal{G}$, is an infinite group (it contains an infinite number of symmetry operations). If we choose, among the operations of $\mathcal{G}$, those that correspond to pure translations, we get the translation subgroup, $T(\mathcal{G})$, which expresses the translational symmetry of the crystal structure. The operations of $T(\mathcal{G})$ are represented by matrix-vector pairs $(\mathbf{I}, \mathbf{t})$ in which the linear (matrix) part $\mathbf{I}$ is always the identity, whereas the

\footnotetext{
${ }^{1}$ Point groups in a low-dimensional space $\mathcal{G}_{0}^{N<3}$, line groups $\mathcal{G}_{1}^{1}$, plane groups $\mathcal{G}_{2}^{2}$ and frieze groups $\mathcal{G}_{1}^{2}$ play a fundamental role in the description of crystal patterns used, for example, in ornaments, but have limited applications to real crystal structures.
}

translation (vector) part $\mathbf{t}$ is always integral if the unit cell chosen to describe the structure is primitive, or can take rational or integral components if it is centred. By applying the operations (I, t) to a point in space chosen as the origin, we get an infinite collection of points distributed in a uniform and periodic way that represents the nodes of the Bravais lattice $\mathbf{L}$ of the structure. Accordingly, in the following we can alternatively write $T(\mathcal{G})$ as $T(\mathbf{L})$, depending on whether we refer to the structure or the lattice, i.e. on whether the group built by the translation operations is regarded as the translation subgroup of the space group $\mathcal{G}$ or the translation group of the lattice $\mathbf{L}$.

If we now take a subgroup $T^{\prime}(\mathcal{G})$ of $T(\mathcal{G})$, i.e. we 'forget' some of the operations $(\mathbf{I}, \mathbf{t})$ of $T(\mathcal{G})$ in such a way that the remaining operations still form a group, the result is a sublattice $\mathbf{L}^{\prime}$. The primitive unit cell of this sublattice $\mathbf{L}^{\prime}$ has a larger volume with respect to the primitive unit cell of the original lattice $\mathbf{L}$ and is therefore a supercell. ${ }^{2}$ The structure corresponding to this supercell is a superstructure (Buerger, 1947). ${ }^{3}$ Quite obviously, by inverting the perspective, $T(\mathcal{G})$ is a supergroup of $T^{\prime}(\mathcal{G})$ and therefore $\mathbf{L}$ is a superlattice of $\mathbf{L}^{\prime}$; its primitive unit cell has a smaller volume than the primitive unit cell of $\mathbf{L}^{\prime}$ and is therefore a subcell. Unfortunately, the correspondence sublattice $\leftrightarrow$ supercell and superlattice $\leftrightarrow$ subcell is often overlooked in the literature, and it is not rare to read about 'superlattices' built on 'supercells' or of 'sublattices' built on 'subcells', which contradicts the concept and definition of super/sublattice.

The confusion is perhaps less surprising if we consider that a sublattice in a space corresponds to a superlattice in the dual space and vice versa. For example, missing some weak reflections in a diffraction pattern leads to choosing a larger cell (supercell) and therefore a (reciprocal) sublattice. This corresponds, in direct space, to choosing a smaller cell (subcell) and therefore a superlattice. Vice versa, extra reflections with respect to those of the basic structure appear in the diffraction pattern of a superstructure, which are known as superstructure reflections (Müller, 2013). Indeed, a superstructure has a larger unit cell (supercell) in direct space, which corresponds to a smaller unit cell (subcell) in reciprocal space and therefore a reciprocal superlattice. One should be careful not to confuse these terms but instead to be quite explicit in the description of the use of 'sub'/super' with respect to the space one is dealing with.

The misunderstanding of the fundamental difference between a lattice and a structure can lead to far more serious consequences, like the extravagant introduction of two additional three-dimensional lattices to account for the hexagonal close-packed structure, misinterpreted as a lattice, and a

\footnotetext{
${ }^{2}$ We make reference to the primitive unit cell of both lattices, which is not necessarily the conventional one, to make the comparison unambiguous. In fact, the conventional unit cell of the two lattices may have a different multiplicity and in the comparison the volume ratio has to be scaled by the multiplicity ratio. Using a primitive cell avoids this additional term.

${ }^{3}$ If a structure is obtained by reducing the symmetry of the original (basic) structure while keeping all the lattice translations, it is called a derivative structure (Buerger, 1947). Its space group is a translationengleiche subgroup of the space group of the basic structure (Müller, 2013).
} 
hypothetical 'rhombohedral close-packed lattice', which is nothing else than a face-centred cubic lattice described by its primitive rhombohedral cell, as shown by the cell parameter relations $a=b=c, \alpha=\beta=\gamma=60^{\circ}$ (Wahab \& Wahab, 2015).

Much more surprising is the confusion between the concepts of lattice and structure. As we have just seen, the former corresponds to the periodicity of the latter. Nevertheless, in the literature the confusion between these two fundamentally different concepts is far from rare. As an example, we can quote the use of 'sphalerite lattice' instead of 'sphalerite structure' (e.g. Sarvaramini et al., 2016). Sphalerite is the polytype of $\mathrm{ZnS}$ stable at ambient conditions; it crystallizes in a space group of type $\overline{4} \overline{3} \mathrm{~m}$ with $\mathrm{Zn}$ in position $4 a$ and $\mathrm{S}$ in position $4 c$. The lattice of sphalerite is face-centred cubic; the structure contains two atoms in the asymmetric unit, i.e. is built by two crystallographic orbits, and is obviously not a lattice. Overlooking the fundamental difference between a crystal structure and its lattice leads to even more severe misunderstanding, like the appearance of terms like 'lattice engineering' (Lai et al., or 2019), as if one were trying to tweak an abstract collection of geometric points instead of a network of atoms.

\section{Crystallographic orbits and Wyckoff positions}

A crystal structure $X$ can be seen as the set-theoretical union of its crystallographic orbits $O_{i}: X=\cup_{i} O_{i}$. The space group $\mathcal{G}$ of the structure $X$ is the set-theoretical intersection of the eigensymmetries $\mathscr{E}_{i}$ of those crystallographic orbits: $\mathcal{G}=\cap_{i} \mathscr{E}_{i}$. Accordingly, the eigensymmetry $\mathscr{E}$ of each crystallographic orbit $O$ can either coincide with the space group $(\mathscr{E}=\mathcal{G})$ or be higher than it (a supergroup: $\mathscr{E} \supset \mathcal{G}$ ), but can never be lower (a subgroup). Depending on whether $\mathscr{E}_{i}=\mathcal{G}$ or $\mathscr{E}_{i} \supset \mathcal{G}$, the $i$ th orbit $O_{i}$ is called characteristic or non-characteristic, respectively. A special case of non-characteristic orbit occurs when the translation subgroup of $\mathscr{E}_{i}$ is a supergroup of the translation subgroup of $\mathcal{G}: T\left(\mathscr{E}_{i}\right) \supset T(\mathcal{G})$. The orbit $O_{i}$ is then called extraordinary (Engel et al., 1984). If none of the orbits $O_{i}$ is extraordinary, then the translation subgroup $T(\mathcal{G})$ of the space group $\mathcal{G}$ is common to all the crystallographic orbits building the crystal structure $X$.

The term crystallographic orbit is relatively poorly known to experimental crystallographers, whereas the term Wyckoff position is used on a daily basis; the two concepts are nevertheless closely related. A crystallographic orbit is an infinite set of points generated from a set of Wyckoff positions if any general coordinate $x, y$ or $z$ that appears in the Wyckoff position is replaced by a specific numerical value. In fact, the concept of crystallographic orbit is more straightforward to define than that of Wyckoff position. If one takes a single point in the asymmetric unit and applies to its fractional coordinates all the infinitely many operations of a space group $\mathcal{G}$, one obtains an infinite set of points that are all equivalent (because related by the operations of $\mathcal{G}$ ). This infinite set of points constitutes a crystallographic orbit. One then assigns the crystallographic orbit to a Wyckoff position in $\mathcal{G}$, on the basis of the site-symmetry groups (notice the plural) $\mathcal{S}$ of points that belong to the crystallographic orbit (notice the singular). A site-symmetry group of a certain position (site) with coordinates $x, y, z$ is the subset of operations of $\mathcal{G}$ (i.e. a subgroup of $\mathcal{G}$ ) which leaves $x, y, z$ invariant (fixed in space). In other words, if $(\mathbf{W}, \mathbf{w})$ is the matrix-column representation of the operations of $\mathcal{G}$, then those operations which satisfy the relation $(\mathbf{W}, \mathbf{w}) \mid x y z)=\mid x y z)^{\mathbf{4}}$ build up the site-symmetry group of the position $x, y, z .(\mathbf{W}, \mathbf{w})$ is composed of a linear (matrix) part $\mathbf{W}$, which represents the rotation, reflection or (roto)inversion component of the operation, and a translation (vector) part $\mathbf{w}$, which is non-zero if the symmetry element does not pass through the origin; it does not include any screw or glide component, in the presence of which no fixed point would exist. The site-symmetry group is then represented by an oriented symbol which corresponds to the linear part $\mathbf{W}$ and is therefore isomorphic (i.e. in 1:1 correspondence) with a crystallographic point group. For example, an atom with coordinates $0, \frac{1}{2}, \frac{1}{2}$ in a space group of type $\mathrm{Cmcm}$ belongs to the Wyckoff position $4 b$; by applying the operations of $\mathrm{Cmcm}$ to the position with coordinates $0, \frac{1}{2}, \frac{1}{2}$ one gets an infinite number of symmetrically equivalent positions with coordinates $0, \frac{1}{2}, \frac{1}{2}$; $0, \frac{1}{2}, 0 ; \frac{1}{2}, 0, \frac{1}{2} ; \frac{1}{2}, 0,0 ; 1, \frac{1}{2}, \frac{1}{2}, 1, \frac{1}{2}, 1 ; \frac{1}{2}, 1, \frac{1}{2} ; \frac{1}{2}, 1,1$ etc. This infinite set of positions can be succinctly represented as $p, q+\frac{1}{2}, r+\frac{1}{2} ; p, q$ $+\frac{1}{2}, r ; p+\frac{1}{2}, q, r+\frac{1}{2} ; p+\frac{1}{2}, q, r$, where $p, q, r$ are integers spanning the interval $-\infty$ to $+\infty$. All these positions belong to one crystallographic orbit, because they are all related by the symmetry operations of $\mathrm{Cmcm}$. If now we chose one position $x, y, z$, corresponding to certain values of $p, q, r$, from the infinitely many positions belonging to this crystallographic orbit, we can select, among the infinitely many symmetry operations of $\mathrm{Cmcm}$, a small finite number which leaves that position invariant; the set of operations selected in this way constitutes the site-symmetry group $\mathcal{S}$ of that position. If we then choose another, different position $x^{\prime}, y^{\prime}, z^{\prime}$, corresponding to different values of $p, q, r$, from the same orbit and repeat the procedure, we obtain a different set of operations of $\mathrm{Cmcm}$ which leaves invariant $x^{\prime}, y^{\prime}, z^{\prime}$; these operations constitute the site-symmetry group $\mathcal{S}^{\prime}$ of $x^{\prime}, y^{\prime}, z^{\prime} . \mathcal{S}$ and $\mathcal{S}^{\prime}$ are, in general, different groups, whose operations share the same linear part $\mathbf{W}$; they differ in their translation part $\mathbf{w}$ because the location of the corresponding symmetry elements is different (otherwise $x, y, z$ and $x^{\prime}, y^{\prime}, z^{\prime}$ would coincide, contrary to the hypothesis). $\mathcal{S}$ and $\mathcal{S}^{\prime}$ are, however, tightly related. In fact, because $x, y, z$ and $x^{\prime}, y^{\prime}, z^{\prime}$ belong to the same crystallographic orbit, there are some operations of the space group that map one position onto the other. Let us select $g$ as one of these operations. The transformation can be written in the following way:

$$
\left.\left.\left.g \mid x, y, z)=\mid x^{\prime}, y^{\prime}, z^{\prime}\right) ; \quad g^{-1} \mid x^{\prime}, y^{\prime}, z^{\prime}\right)=\mid x, y, z\right) .
$$

Each of these positions is invariant under the operations of the corresponding site-symmetry group. Therefore,

$$
\left.\left.\mathcal{S} \mid x, y, z)=\mid x, y, z) ; \quad \mathcal{S}^{\prime} \mid x^{\prime}, y^{\prime}, z^{\prime}\right)=\mid x^{\prime}, y^{\prime}, z^{\prime}\right) .
$$

If we insert (2) into (1) we obtain

${ }^{4}$ We use here the notation $\left.\mid x y z\right)$ to indicate a column matrix. 


$$
\begin{aligned}
g \mid x, y, z) & \left.=g \mathcal{S} \mid x, y, z)=g \mathcal{S} g^{-1} \mid x^{\prime}, y^{\prime}, z^{\prime}\right) \\
& \left.\left.=\mid x^{\prime}, y^{\prime}, z^{\prime}\right)=\mathcal{S}^{\prime} \mid x^{\prime}, y^{\prime}, z^{\prime}\right),
\end{aligned}
$$

from which we obtain

$$
\mathcal{S}^{\prime}=g S g^{-1}
$$

The transformation (4) is a similarity transformation known as conjugation. We have just shown that the positions $x, y, z$ and $x^{\prime}, y^{\prime}, z^{\prime}$ are transformed directly from one to the other under the action of the operation $g$, whereas the sitesymmetry groups of those positions $\mathcal{S}$ and $\mathcal{S}^{\prime}$ are transformed by conjugation. To say that two positions are related by a space-group operation is therefore equivalent to saying that their site-symmetry groups are conjugate under that operation.

One may therefore get the impression that Wyckoff positions and crystallographic orbits are the same thing, simply defined differently. This is only true for the positions like $4 b$ in $\mathrm{Cmcm}$ discussed above, which do not have any free coordinates; in such a case, only one crystallographic orbit is assigned to the Wyckoff position. If instead at least one fractional coordinate is of general type, then one gets an infinite number of crystallographic orbits, one for any possible fractional value of the general coordinate. For example, in a space group of type $\mathrm{Cmcm}$, a position of type $0, y, \frac{1}{4}$ corresponds to an infinite number of crystallographic orbits, one for each value of $y$, and they are all assigned to the same Wyckoff position, namely $4 c$, because their site-symmetry groups are all of type $m 2 m$ and they are all conjugate under the operations of $\mathrm{Cmcm}$. A position of type $x, y, \frac{1}{4}$, again in $C m c m$, also corresponds to an infinite number of crystallographic orbits, one for each value of $x$ and $y$ with the exception of $x=0$; they are all assigned to the same Wyckoff position, namely $8 g$, because their sitesymmetry groups are all of type ..m and they are all conjugate under the operations of $C m c m$. Therefore, there exists in general a correspondence $\infty: 1$ from crystallographic orbits to Wyckoff positions. This distinction between crystallographic orbits and Wyckoff positions is fundamental for the following discussion, in particular for the definition of a substructure.

\section{Sublattice versus substructure}

It is not uncommon to read the term 'sublattice' used to indicate a single crystallographic orbit. For example, the doping of $\mathrm{ZnS}$ by $\mathrm{CuCl}$ is described as ' $\mathrm{Cu}^{+}$cations occupying the $\mathrm{Zn}$ sublattice', paired with the 'incorporation of $\mathrm{Cl}^{-}$ anions in the sulfur sublattice' (Kryshtab et al., 2006). As we have shown above, a sublattice is a lattice (not a structure) $\mathbf{L}^{\prime}$ which is obtained from another (parent) lattice $\mathbf{L}$ by removing a subset of the translation operations, so that the remaining translation operations still form a group. This translation group $T\left(\mathbf{L}^{\prime}\right)$ is a subgroup of the translation group $T(\mathbf{L})$ of the parent lattice, which is expressed as $T\left(\mathbf{L}^{\prime}\right) \subset T(\mathbf{L})$. We have also seen that the structure of an ideal crystal is the settheoretical union of a finite number of crystallographic orbits, and that the translation subgroup of the $i$ th crystallographic orbit, $T\left(\mathscr{E}_{i}\right)$, either coincides with that of the whole structure, i.e. $T\left(\mathscr{E}_{i}\right)=T(\mathcal{G})$ (in the case of characteristic orbits and of non-characteristic but not extraordinary orbits) or is a supergroup of it, i.e. $T\left(\mathscr{E}_{i}\right) \supset T(\mathcal{G})$ (in the case of extraordinary orbits). Therefore, the lattice defined by the translation operations of a single crystallographic orbit can either coincide with the lattice of the whole crystal structure or be a superlattice of it: certainly not a sublattice. The same conclusion is obtained when considering a set consisting of more than one crystallographic orbit, which still is a subset of the whole crystal structure. In fact, the translation vectors common to this subset of orbits can never result in a subgroup of $T(\mathcal{G})$. This is expressed formally as $\cap_{i} T\left(\mathscr{E}_{i}\right) \supseteq T(\mathcal{G})$, where the $\supset$ (supergroup) can be realized under the necessary (but

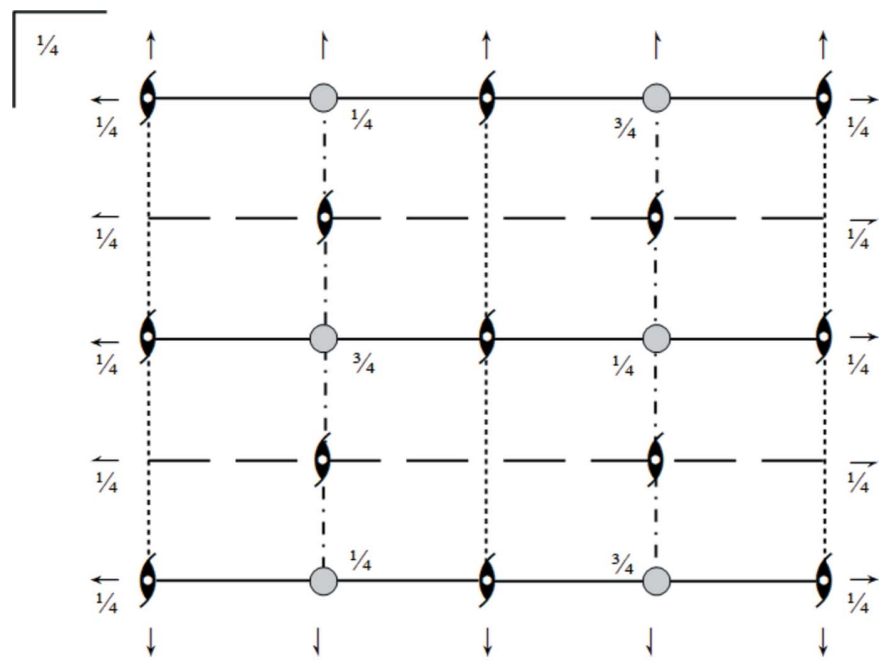

(a)

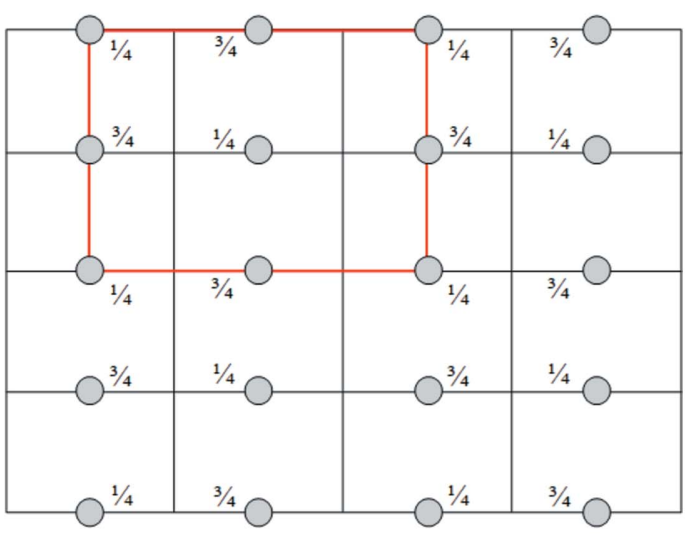

(b)

Figure 1

The crystallographic orbit generated by the position $0, \frac{1}{4}, \frac{1}{4}$ in a space group of type $\mathrm{Cmcm}$, seen in projection along the [001] axis. This crystallographic orbit is one of the infinitely many that belong to the Wyckoff position $4 c\left(0, y, \frac{1}{4}\right)$; the particular orbit shown has $y=\frac{1}{4} \cdot(a)$ Projection of the symmetry elements and of the atomic positions, in grey. One unit cell is shown. $(b)$ Projection of four unit cells, from which the symmetry elements have been removed. The atoms occur alternately with $z=\frac{1}{4}$ and $z=\frac{3}{4}$, as shown in the figure. The eigensymmetry of this crystallographic orbit is Fmmm and its unit cell is shown in red, with the origin moved onto one atom for ease of visualization. The basis vectors are parallel to those of the space group and have the same length, but the unit cell is $F$ centred. 
not sufficient) conditions that (1) the running index spans a subset of the crystallographic orbits building the crystal structure and (2) these crystallographic orbits are all extraordinary orbits. The conditions are not sufficient because the intersection $\cap_{i} T\left(\mathscr{E}_{\mathrm{i}}\right)$ is not necessarily a supergroup of $T(\mathcal{G})$. For example, in a space group of type $\mathrm{Cmcm}$, the crystallographic orbit generated by the position $0, \frac{1}{4}$, $\frac{1}{4}$, which corresponds to the Wyckoff position $4 c$ for $y=\frac{1}{4}$, is extraordinary, with eigensymmetry Fmmm and basis vectors in common with those of the space group (Fig. 1). In the same space group, the crystallographic orbit generated by the position $0, \frac{1}{2}, 0$ is the only crystallographic orbit corresponding to the Wyckoff position $4 b$ and is extraordinary, with eigensymmetry $C \mathrm{mmm}$ and basis vectors $\mathbf{a}, \mathbf{b}, \mathbf{c} / 2$ with respect to those of $\mathrm{Cmcm}$

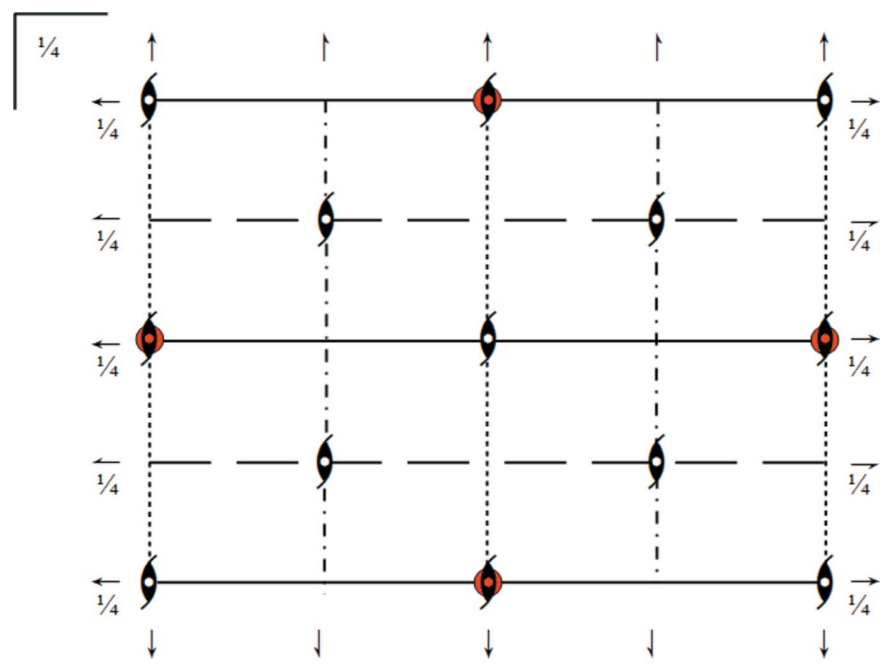

(a)

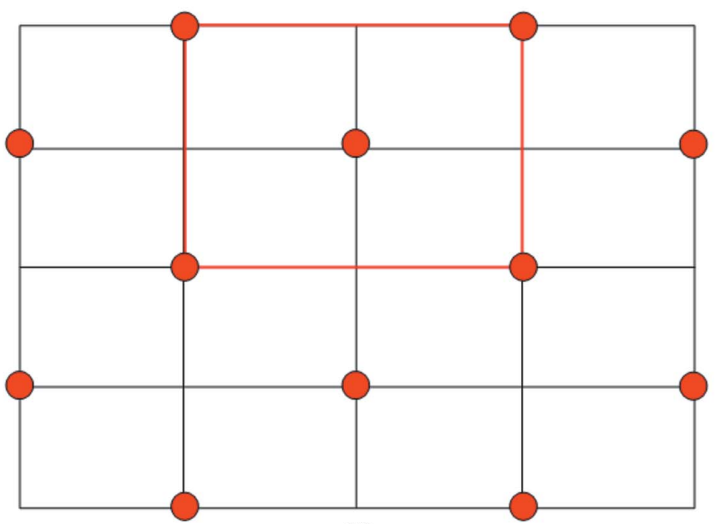

(b)

Figure 2

The crystallographic orbit generated by the position $0, \frac{1}{2}, 0$ in the same space group of type $\mathrm{Cmcm}$ used in Fig. 1, seen in projection along the [001] axis. This crystallographic orbit is the only one that belongs to the Wyckoff position $4 b$. Same conventions as in Fig. 1. All the atoms occur for $z=0$ and $z=\frac{1}{2}$; these two coordinates are not shown in the figure because they are the same for all atoms. In $(a)$, the projected atomic positions (in red) fall on the same poisitons as the symbols of $2_{1}$ screw axes (black). (b) The eigensymmetry of this crystallographic orbit is $\mathrm{Cmmm}$ and its unit cell is shown in red, with the origin moved onto one atom for ease of visualization. The basis vectors are parallel to those of the space group, but the $\mathbf{c}$ basis vector has half length owing to the presence of atoms every half period in the unit cell of $\mathrm{Cmcm}$.
(Fig. 2). If these two orbits are both occupied by atoms, the structure they build up has the same symmetry as the space group, including of course the translation subgroup (Fig. 3).

Concretely, if a crystal structure is composed of $m>1$ crystallographic orbits and one needs to consider $m^{\prime}<m$ crystallographic orbits, these constitute a substructure, not a sublattice. If $m^{\prime}=1$, the substructure actually coincides with a single crystallographic orbit and should be identified in this way.

In the study of magnetic properties of crystals, it is common to write about a 'magnetic sublattice' to indicate the 'array of all magnetic moments of identical ions oriented in one direction' (e.g. Borovik-Romanov et al., 2014). Magnetic moments are associated with atoms, not with lattice nodes. The term 'magnetic sublattice' should therefore be replaced by 'magnetic substructure'.

\section{Dimensionality versus periodicity}

It has unfortunately become quite common to use the term 'low-dimensional materials' to refer to crystalline materials built by subperiodic building blocks. A clear confusion occurs here between the dimensionality of the space in which the material physically exists and the dimensionality of the subspace in which its building modules are periodic. We briefly analyse the three cases corresponding to $\mathcal{G}_{2}^{3}, \mathcal{G}_{1}^{3}$ and $\mathcal{G}_{0}^{3}$.

(1) Diperiodic modules (sheets, layers). Structures consisting of a single layer of atoms are today the object of intensive research because of their importance in applications such as photovoltaics, semiconductors, electrodes and water purification. If the layer is perfectly flat and its two sides in the direction of missing periodicity are absolutely identical (like a sheet of paper with the two faces perfectly identical) then one may agree to ignore the operations turning the layer upside down and describe it as two dimensional; it is nevertheless an approximation corresponding to taking the thickness of the

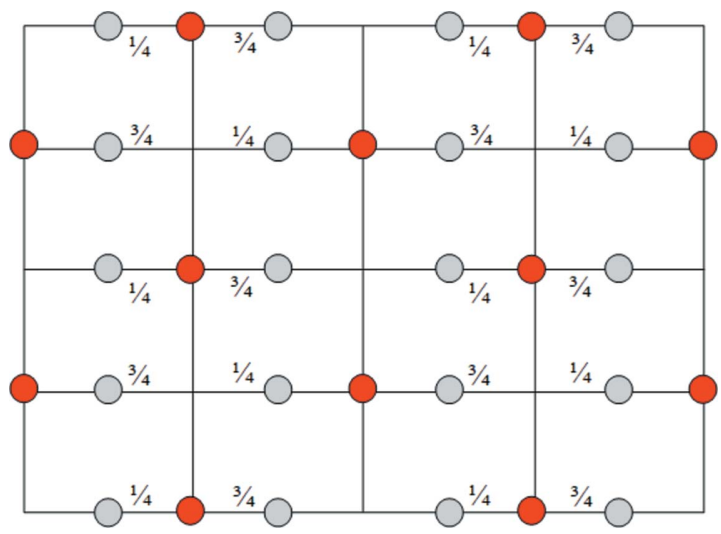

Figure 3

The union (coexistence) of the two crystallographic orbits in Figs. 1 and 2 results in the space group $\mathrm{Cmcm}$ as intersection of the eigensymmetries Fmmm and Cmmm. Although both crystallographic orbits are extraordinary, their union reduces the symmetry of the pattern to that of the space group. 
Table 1

Summary of some common errors found in the crystallographic literature and often used in crystallographic jargon.

\begin{tabular}{|c|c|c|}
\hline Incorrect term or expression & Correct use & Remark \\
\hline $\begin{array}{l}\text { Lattice (when talking about a periodic } \\
\text { distribution of atoms in space) }\end{array}$ & Structure & A lattice is a mathematical abstraction that expresses the periodicity of the structure \\
\hline $\begin{array}{l}\text { Superlattice (when making reference to } \\
\text { the lattice based on a supercell) }\end{array}$ & Sublattice & $\begin{array}{l}\text { The unit cell is larger (supercell) than that of the parent lattice, which means that the } \\
\text { translation group is a subgroup; therefore, the correct term is sublattice }\end{array}$ \\
\hline $\begin{array}{l}\text { Sublattice (when making reference to } \\
\text { the lattice based on a subcell) }\end{array}$ & Superlattice & $\begin{array}{l}\text { The unit cell is smaller (subcell) than that of the parent lattice, which means that the } \\
\text { translation group is a supergroup; therefore, the correct term is superlattice }\end{array}$ \\
\hline $\begin{array}{l}\text { Sublattice (when making reference to } \\
\text { one or more subsets of atoms) }\end{array}$ & Substructure & $\begin{array}{l}\text { If the substructure is built by atoms that are all equivalent under the operations of the space } \\
\text { group, then it consists of just one crystallographic orbit }\end{array}$ \\
\hline
\end{tabular}

layer to zero. This approximation can be justified in materials like graphene. It is however completely unacceptable if the two sides of the layer are not identical (like a sheet of paper with two different colours on the two faces) or for layers whose thickness goes beyond a single atom. A number of examples of incorrect descriptions can be found in the literature, like the '2D hybrid perovskites' which have thicknesses of one to several octahedral sheets, surrounded on both sides by organic molecules (e.g. Jagielski et al., 2017). It should be quite obvious that describing a layer several atoms thick as 'two dimensional' does not make sense. The layer is three dimensional but diperiodic; it cannot be described by any of the 17 types of plane groups $G_{2}^{2}$ but requires one of the 80 types of layer groups $\mathcal{G}_{2}^{3}$.

(2) Monoperiodic modules (chains). Chains of atoms are often incorrectly described as 'mono-dimensional' (e.g. Lhoste et al., 2009). A chain cannot be considered as mono-dimensional and therefore described by one of the two line groups $\mathcal{G}_{1}^{1}$; it is simply a monoperiodic unit in the three-dimensional space, whose description requires one of the $75 \operatorname{rod}$ groups $\mathcal{G}_{1}^{3}$.

(3) Zero-periodic modules (bricks). Structures built of finite building blocks are incorrectly referred to as 'zero dimensional'. For example, Chen et al. (2018) have reported a leadfree hybrid perovskite containing 'zero-dimensional discrete anionic clusters'. A cluster of atoms is obviously three dimensional but zero periodic, i.e. it is a finite building block. Its symmetry is described by a three-dimensional point group $\mathcal{G}_{0}^{3}$.

\section{Conclusions}

The description of crystal structures is of paramount importance in the study of condensed matter, but unfortunately a sort of laboratory jargon has found its way into the literature. A systematic screening of the terminological mistakes would certainly take a much wider space and is beyond the scope of this report (see, however, our previous analyses; Nespolo, $2015,2018)$. We have pointed out some examples that are unfortunately often spotted in contemporary literature, with the hope of helping authors to avoid repeating these errors and students to avoid falling into these terminological traps.
Table 1 gives a summary of the common mistakes that we have pointed out and how to correct them.

\section{Acknowledgements}

Critical remarks by two anonymous reviewers helped in improving the manuscript and are gratefully acknowledged.

\section{References}

Borovik-Romanov, A. S., Grimmer, H. \& Kenzelmann, M. (2014). International Tables for Crystallography, Vol. D, Physical Properties of Crystals, 2nd ed., edited by A. Authier, ch. 1.5. Chichester: Wiley.

Buerger, M. J. (1947). J. Chem. Phys. 15, 1-16.

Chen, W.-J., Chu, K.-B. \& Song, J.-L. (2018). Acta Cryst. C74, 1744 1749.

Conway, J. H. \& Sloane, N. J. A. (1998). Sphere Packings, Lattices and Groups, 3rd ed. New York: Springer.

Devi, L. V., Selvalakshmi, T., Sellaiyan, S., Uedono, A., Sivaji, K. \& Sankar, S. (2017). J. Alloys Compd. 709, 496-504.

Engel, P., Matsumoto, T., Steinmann, G. \& Wondratschek, H. (1984). The Non-characteristic Orbits of the Space Groups, Zeitschrift für Kristallographie Supplemente 1. Berlin: De Gruyter Oldenbourg. Jagielski, J., Kumar, S., Yu, W.-Y. \& Shih, C.-J. (2017). J. Mater. Chem. $C, \mathbf{5}, 5610-5627$.

Kopský, V. \& Litvin, D. B. (2010). Editors. International Tables for Crystallography, Vol. E, Subperiodic Groups, 2nd ed. Chichester: Wiley.

Kryshtab, T., Khomchenko, V. S., Andraca-Adame, J. A., Rodionov, V. E., Khachatryan, V. B. \& Tzyrkunov, Y. A. (2006). Superlattice Microstruct. 40, 651-656.

Lai, Y.-T., Wang, T., O’Dell, S., Louder, M. K., Schön, A., Cheung, C. S. F., Chuang, G.-Y., Druz, A., Lin, B., McKee, K., Peng, D., Yang, Y., Zhang, B., Herschhorn, A., Sodroski, J., Bailer, R. T., DoriaRose, N. A., Mascola, J. R., Langley, D. R. \& Kwong, P. D. (2019). Nat. Commun. 10, 47.

Lhoste, J., Gervier, R., Maisonneuve, V., Leblanc, M. \& Adil, K. (2009). Solid State Sci. 11, 1582-1586.

Lundow, P. H. \& Campbell, I. A. (2018). Physica A, 511, 40-53.

Müller, U. (2013). Symmetry Relationships Between Crystal Structures. IUCr/Oxford Science Publications.

Nespolo, M. (2015). J. Appl. Cryst. 48, 1290-1298.

Nespolo, M. (2018). J. Appl. Cryst. 51, 1221-1225.

Nespolo, M., Ferraris, G., Ďurovič, S. \& Takéuchi, Y. (2004). Z. Kristallogr. 219, 773-778.

Sarvaramini, A., Larachi, F. \& Hart, B. (2016). Appl. Surf. Sci. 367, 459-472.

Wahab, M. A. \& Wahab, K. M. (2015). ISST J. Appl. Phys. 6, 1-10. 